Gilles Aubert Pierre Kornprobst

\title{
Mathematical Problems in Image Processing
}

Partial Differential Equations and the Calculus of Variations

Second Edition 


\section{Contents}

Foreword vii

Preface to the Second Edition xi

Preface to the First Edition $\quad$ xv

Guide to the Main Mathematical Concepts and Their Application $\quad \mathbf{x x v}$

Notation and Symbols $\quad$ xxvii

1 Introduction 1

1.1 The Image Society . . . . . . . . . . . . . . . . . . 1

1.2 What Is a Digital Image? . . . . . . . . . . . . . . . 3

1.3 About Partial Differential Equations (PDEs) . . . . . . 5

1.4 Detailed Plan . . . . . . . . . . . . . . 5

2 Mathematical Preliminaries $\quad 29$

How to Read This Chapter . . . . . . . . . . . . . . . . . . 29

2.1 The Direct Method in the Calculus of Variations . . . . . 30

2.1.1 Topologies on Banach Spaces ... . . . . . . 30

2.1.2 Convexity and Lower Semicontinuity . . . . . . 32

2.1.3 Relaxation ................. 37

2.1.4 About $\Gamma$-Convergence........... . . 40

2.2 The Space of Functions of Bounded Variation . . . . . 42 
2.2.1 Basic Definitions on Measures ......... 43

2.2.2 Definition of $B V(\Omega) \quad \ldots \ldots \ldots . \ldots . \ldots 45$

2.2 .3 Properties of $B V(\Omega) \ldots \ldots \ldots$

2.2.4 Convex Functions of Measures . . . . . . . . . . 50

2.3 Viscosity Solutions in PDEs . . . . . . . . . . . . . . 50

2.3.1 About the Eikonal Equation . . . . . . . . . . . 50

2.3.2 Definition of Viscosity Solutions . . . . . . . . 52

2.3.3 About the Existence ............. . . 54

2.3.4 About the Uniqueness ............ 55

2.4 Elements of Differential Geometry: Curvature . . . . . 57

2.4.1 Parametrized Curves ................. 58

2.4.2 Curves as Isolevel of a Function $u \ldots \ldots$. . . . 58

2.4 .3 Images as Surfaces . . . . . . . . . . . . . . . 59

2.5 Other Classical Results Used in This Book . . . . . . . 60

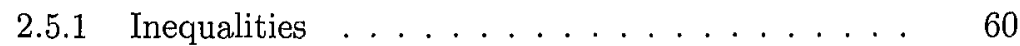

2.5.2 Calculus Facts . . . . . . . . . . . . . 62

2.5.3 About Convolution and Smoothing ....... 62

2.5.4 Uniform Convergence . . . . . . . . . . . . 63

2.5.5 Dominated Convergence Theorem . . . . . . . . 64

2.5.6 Well-Posed Problems . . . . . . . . . . . . . 64

3 Image Restoration $\quad 65$

How to Read This Chapter . . . . . . . . . . . . . 65

3.1 Image Degradation ............... 66

3.2 The Energy Method .......................... 68

3.2.1 An Inverse Problem . . . . . . . . . . . . . . . 68

3.2.2 Regularization of the Problem .......... . 69

3.2.3 Existence and Uniqueness of a Solution for

the Minimization Problem . . . . . . . . . . 72

3.2.4 Toward the Numerical Approximation . . . . . 76

The Projection Approach . . . . . . . . . 76

The Half-Quadratic Minimization Approach . . . 79

3.2.5 Some Invariances and the Role of $\lambda \ldots$. . . . . . 87

3.2.6 Some Remarks on the Nonconvex Case . . . . . . 90

3.3 PDE-Based Methods ... . . . . . . . . . . . 94

3.3.1 Smoothing PDEs . . . . . . . . . . . . . . . 95

The Heat Equation . . . . . . . . . . . . . . . . . 95

Nonlinear Diffusion . . . . . . . . . . . . 98

The Alvarez-Guichard-Lions-Morel

Scale Space Theory . . . . . . . . . . . . . . . 107

Weickert's Approach . . . . . . . . . . . . 113

Surface Based Approaches . . . . . . . . . . . . 117

3.3.2 Smoothing-Enhancing PDEs . . . . . . . . . . 121

The Perona and Malik Model . . . . . . . . 121 
Regularization of the Perona and Malik Model:

Catté et al. . . . . . . . . . . . . . . . . 123

3.3.3 Enhancing PDEs ... . . . . . . . . . . 128

The Osher and Rudin Shock Filters . . . . . . . . 128

A Case Study: Construction of a Solution by

the Method of Characteristics . . . . . . . . 130

Comments on the Shock-Filter Equation . . . . . 134

3.3.4 Neighborhood Filters, Nonlocal Means Algorithm, and PDEs . . . . . . . . . . . . . 137

Neighborhood Filters . . . . . . . . . . . . 138

How to Suppress the Staircase Effect? . . . . . . 143

Nonlocal Means Filter (NL-Means) . . . . . . . 146

4 The Segmentation Problem 149

How to Read This Chapter . . . . . . . . . . . . . . . . 149

4.1 Definition and Objectives ............... 150

4.2 The Mumford and Shah Functional . . . . . . . . . 153

4.2.1 A Minimization Problem . . . . . . . . . 153

4.2.2 The Mathematical Framework for the

Existence of a Solution . . . . . . . . . . . . . . 154

4.2.3 Regularity of the Edge Set . . . . . . . . . . 162

4.2.4 Approximations of the Mumford and Shah

Functional ........................ 166

4.2.5 Experimental Results . . . . . . . . . . . 171

4.3 Geodesic Active Contours and the Level-Set Method. . . 173

4.3.1 The Kass-Witkin-Terzopoulos model . . . . . . . 173

4.3.2 The Geodesic Active Contours Model . . . . . . . 175

4.3 .3 The Level-Set Method . . . . . . . . . . . . . . . 182

4.3.4 The Reinitialization Equation . . . . . . . . . . 194

Characterization of the Distance Function . . . . 195

Existence and Uniqueness . . . . . . . . . . . 198

4.3.5 Experimental Results . . . . . . . . . . . . 206

4.3.6 About Some Recent Advances . . . . . . . . . . 208

Global Stopping Criterion . . . . . . . . . . 208

Toward More General Shape Representation . . . 211

5 Other Challenging Applications 213

How to Read This Chapter . . . . . . . . . . . . . . 213

5.1 Reinventing Some Image Parts by Inpainting . . . . . . . 215

5.1 .1 Introduction . . . . . . . . . . . . . 215

5.1 .2 Variational Models .............. 216

The Masnou and Morel Approach . . . . . . . . 216

The Ballester et al. Approach . . . . . . . . . 218

The Chan and Shen Total Variation Minimization

Approach ............ 220 
5.1.3 PDE-Based Approaches . . . . . . . . . . . 222

The Bertalmio et al. Approach . . . . . . . . . . 223

The Chan and Shen Curvature-Driven Diffusion Approach . . . . . . . . . 224

5.1.4 Discussion . . . . . . . . . . . . . . . . . 225

5.2 Decomposing an Image into Geometry and Texture ... 228

5.2 .1 Introduction . . . . . . . . . . . . . . . 228

5.2.2 A Space for Modeling Oscillating Patterns . . . . 229

5.2 .3 Meyer's Model . . . . . . . . . . . . . . . . 232

5.2.4 An Algorithm to Solve Meyer's Model . . . . . 233

Prior Numerical Contribution . . . . . . . . . . 234

The Aujol et al. Approach . . . . . . . . . . . . . 234

Study of the Asymptotic Case . . . . . . . . . . 241

Back to Meyer's Model . . . . . . . . . . . . . 242

5.2.5 Experimental Results . . . . . . . . . . . . . . . 245

Denoising Capabilities . . . . . . . . . . 245

Dealing With Texture . . . . . . . . . . . . 248

5.2.6 About Some Recent Advances . . . . . . . . . . . 248

5.3 Sequence Analysis . . . . . . . . . . . . . . . . . . . . 249

5.3 .1 Introduction . . . . . . . . . . . . . . . . . . 249

5.3.2 The Optical Flow: An Apparent Motion . . . . . 250

The Optical Flow Constraint (OFC) . . . . . . 252

Solving the Aperture Problem . . . . . . . . . 253

Overview of a Discontinuity-Preserving

Variational Approach . . . . . . . . . . . . . 256

Alternatives to the OFC . . . . . . . . . 260

5.3.3 Sequence Segmentation . . . . . . . . . . . . 261

Introduction . . . . . . . . . . . . . . 261

A Variational Formulation . . . . . . . . . . . 264

Mathematical Study of the

Time-Sampled Energy . . . . . . . . . . . 265

Experiments . . . . . . . . . . . . . . . . 269

5.3.4 Sequence Restoration . . . . . . . . . . . . . 271

Principles of Video Inpainting . . . . . . . . . . 276

Total Variation (TV) Minimization Approach . . 277

Motion Compensated (MC) Inpainting . . . . . . 277

5.4 Image Classification . . . . . . . . . . . . . . . . . . . . 281

5.4 .1 Introduction . . . . . . . . . . . . . . . . . 281

5.4.2 A Level-Set Approach for

Image Classification . . . . . . . . . . . . . . 282

5.4.3 A Variational Model for Image Classification and Restoration . . . . . . . . . . . . . . 290

5.5 Vector-Valued Images . . . . . . . . . . . . . . . . . . . 299

$5.5 .1 \quad$ Introduction . . . . . . . . . . . . . . . . . 299

5.5.2 An Extended Notion of Gradient . . . . . . . 300 
5.5.3 The Energy Method . . . . . . . . . . . . . . . 300

5.5.4 PDE-Based Methods . . . . . . . . . . . . . . 302

A Introduction to Finite Difference Methods 307

How to Read This Chapter . . . . . . . . . . . . . . 307

A.1 Definitions and Theoretical Considerations Illustrated

by the 1-D Parabolic Heat Equation . . . . . . . . . . 308

A.1.1 Getting Started . . . . . . . . . . . . . . . . 308

A.1.2 Convergence . . . . . . . . . . . . . . 311

A.1.3 The Lax Theorem . . . . . . . . . . . . . . . 313

A.1.4 Consistency . . . . . . . . . . . . . . . . . . . 313

A.1.5 Stability . . . . . . . . . . . . . . 315

A.2 Hyperbolic Equations . . . . . . . . . . . . . . 320

A.3 Difference Schemes in Image Analysis . . . . . . . . . . 329

A.3.1 Getting Started . . . . . . . . . . . . . 329

A.3.2 Image Restoration by Energy Minimization . . . 333

A.3.3 Image Enhancement by the Osher and Rudin

Shock Filters . . . . . . . . . . . . 336

A.3.4 Curve Evolution with the Level-Set Method . . . 338

Mean Curvature Motion . . . . . . . . . . . 339

Constant Speed Evolution . . . . . . . . . . . 340

The Pure Advection Equation . . . . . . . . . . . 341

Image Segmentation by the Geodesic

Active Contour Model . . . . . . . . . . . . 342

B Experiment Yourself! 343

How to Read This Chapter . . . . . . . . . . . . . . . . 343

B.1 The CImg Library . . . . . . . . . . . . . . . . . . . . . 344

B.2 What Is Available Online? . . . . . . . . . . . . . . 344

$\begin{array}{ll}\text { References } & 349\end{array}$

$\begin{array}{ll}\text { Index } & \mathbf{3 7 3}\end{array}$ 